\title{
Effect of a 12-Week Summer Break on School Day Physical Activity and Health-Related Fitness in Low-Income Children from CSPAP Schools
}

\author{
You Fu, ${ }^{1}$ Timothy A. Brusseau, ${ }^{2}$ James C. Hannon, ${ }^{3}$ and Ryan D. Burns ${ }^{2}$ \\ ${ }^{1}$ University of Nevada, 1664 North Virginia Street, Reno, NV 89557, USA \\ ${ }^{2}$ University of Utah, 250 S. 1850 E., Salt Lake City, UT 84112, USA \\ ${ }^{3}$ West Virginia University, 375 Birch St., Morgantown, WV 26505, USA \\ Correspondence should be addressed to You Fu; fuyou1983@gmail.com
}

Received 14 December 2016; Accepted 26 February 2017; Published 9 March 2017

Academic Editor: David Strogatz

Copyright (C) 2017 You Fu et al. This is an open access article distributed under the Creative Commons Attribution License, which permits unrestricted use, distribution, and reproduction in any medium, provided the original work is properly cited.

Background. The purpose of this study was to examine the effect of a 12-week summer break on school day physical activity and health-related fitness (HRF) in children from schools receiving a Comprehensive School Physical Activity Program (CSPAP). Methods. Participants were school-aged children $(N=1,232 ; 624$ girls and 608 boys; mean age $=9.5 \pm 1.8$ years $)$ recruited from three low-income schools receiving a CSPAP. Physical activity and HRF levels were collected during the end of spring semester 2015 and again during the beginning of fall semester 2015. Physical activity was assessed using the Yamax DigiWalker CW600 pedometer. HRF measures consisted of body mass index (BMI) and the Progressive Aerobic Cardiovascular Endurance Run (PACER). Results. Results from a doubly MANCOVA analysis indicated that pedometer step counts decreased from 4,929 steps in the spring to 4,445 steps in the fall (mean difference $=484$ steps; $P<0.001$; Cohen's $d=0.30$ ) and PACER laps decreased from 31.2 laps in the spring to 25.8 laps in the fall (mean difference $=5.4$ laps; $P<0.001$; Cohen's $d=0.33$ ). Conclusions. Children from schools receiving a CSPAP intervention had lower levels of school day physical activity and cardiorespiratory endurance following a 12-week summer break.

\section{Introduction}

Despite the numerous benefits of meeting recommended levels of physical activity [1-3], a majority of children are not meeting 60 minutes of moderate-to-vigorous physical activity (MVPA) per day [3, 4]. Recently, the Institutes of Medicine has recommended at least one-half of the recommended levels of MVPA should be achieved during school hours [5]. Because the majority of school day is spent in sedentary behaviors, achieving at least 30 minutes of MVPA during school hours presents a challenge. The Centers for Disease Control and Prevention has recommended that schools adopt Comprehensive School Physical Activity Programming (CSPAP) to facilitate achievement of school day MVPA [6]. CSPAP is a multicomponent approach in which schools use all available resources to improve the physical activity behaviors of children [6]. Multicomponent school-based interventions have been shown to be effective in promoting school day physical activity during the school year $[7,8]$; however the long-term sustainability and efficacy of this behavioral model are questionable.

Although the goal of CSPAP is to improve MVPA levels in children, it is the physiological trait of having healthy levels of body composition and/or higher levels of cardiorespiratory endurance that has a stronger protective effect on developing cardiometabolic disease risk factors in the pediatric population $[9,10]$. Over the past couple of decades, there has been an emphasis placed on improving health via increasing health-related fitness (HRF) levels in children [11]. The five domains of HRF include body composition, cardiorespiratory endurance, muscular strength and endurance, and flexibility [11]. Of these five domains, body composition and cardiorespiratory endurance have the strongest links to health outcomes $[12,13]$. Therefore, studies aiming to examine the 
efficacy of school-based physical activity interventions like CSPAP should also analyze change in HRF levels.

All children can benefit from optimal levels of physical activity and HRF; however low-income children may especially benefit $[14,15]$. Low-income and/or disadvantaged children have relatively fewer opportunities to participate in free-living physical activity during the day, have poor built-in environments conductive for physical activity participation, and, if of an ethnic minority, have a greater prevalence of unfavorable cardiometabolic biomarkers compared to nonHispanic Caucasian children and/or children of a higher socioeconomic classification [16-18]. Indeed, it has been shown that low socioeconomic status children display lower levels of MVPA compared to higher socioeconomic status children [19]. Therefore, school-based interventions like CSPAP have the potential to greatly benefit this specific pediatric population.

Despite the potential benefits of programs like CSPAP, its long-term efficacy has not been established. A time period that may attenuate the potential benefits gained from a CSPAP intervention is during summer break. Although most studies show that physical activity behaviors are the highest during the summer months in children, some studies have indicated that physical activity is compromised during the summer, possibly because of weather patterns displaying outside temperatures that are too hot and uncomfortable [20], typical to summer temperatures recorded in the geographic regions of the South and Southwestern US [21]. Declines in physical activity during the summer months in these geographic regions may contribute to decreases in HRF levels. This phenomenon may be more prevalent in children from low-income families because of the factors that were stated previously. Thus, the benefits of the CSPAP on children may have the potential to be lost during a long break between school sessions. Despite this possibility, no research has examined the influence that a summer break has on physical activity and HRF levels in low-income children who are enrolled in schools receiving CSPAP. Therefore, the purpose of this study was to examine the effect of a 12-week summer break on physical activity and HRF in children from schools receiving a CSPAP intervention. It was hypothesized that the children's physical activity and HRF levels will decrease from the end of spring semester to the beginning of fall semester.

\section{Methods}

2.1. Participants. Participants were a convenience sample of 1,232 school-aged children (624 girls and 608 boys; mean age $=9.5 \pm 1.8$ years) recruited from three low-income elementary schools receiving government financial assistance (i.e., "Title I Schools") from the Mountain West Region of the US. The schools were receiving a CSPAP intervention during the time of data collection. The CSPAP at each of the three schools was for three years. The data used in this study consisted of measures collected at the end of the first CSPAP year and the beginning of the second CSPAP year. Children were recruited from the 1st to 6th grades. Approximately $60.6 \%$ of the sample was of Hispanic/Latino ethnicity, $13.7 \%$ was Pacific Islander, $10.0 \%$ was Caucasian, $7.8 \%$ was African American, 3.5\% was Asian, and approximately $4.0 \%$ was classified as other. Written assent was obtained from the students and consent was obtained from the parents prior to data collection. The University Institutional Review Board approved the protocols employed in this study.

\subsection{Instrumentation}

2.2.1. Physical Activity. Physical activity was measured using Yamax DigiWalker CW600 pedometers (Tokyo, Japan). Each student in the sample $(N=1,232)$ wore a pedometer for one school week. The pedometers were worn for 5 school days (Monday through Friday) between the hours of 8 a.m. and 3 p.m. on the right hip at the level of the iliac crest in line with the right knee. Classroom teachers, physical educators, and members of the research team ensured that the devices were worn during the entirety of the school day.

The pedometers included a seven-day memory that was used to record steps each day of the school week. Yamax DigiWalker models have been shown to be a valid measure for physical activity in children [22]. The Yamax DigiWalker pedometers have also been shown to provide a reliable estimate of step counts in elementary school-aged children [23]. Participants were included in the analysis if they had recorded data for at least 3 valid days of the school week, to ensure that the devices were worn for the majority of the school week, and had valid data at both spring and fall timepoints $(1,232 / 1,260 ; 97.7 \%)$.

2.2.2. Health-Related Fitness. Body mass index (BMI) was calculated using standard procedures taking a student's weight in kilograms divided by the square or his or her height in meters. Height was measured to the nearest 0.01 meters using a portable stadiometer (Seca 213; Hanover, MD, USA) and weight was measured to the nearest 0.1 kilograms using a portable medical scale (BD-590; Tokyo, Japan). Height and weight were collected in a private room during each student's physical education class.

Cardiorespiratory endurance was measured using the 20-meter Progressive Aerobic Cardiovascular Endurance Run (PACER), administered during each student's physical education class. The PACER was conducted on a marked gymnasium floor with background music provided by a compact disc. Each student was instructed to run from one floor marker to another floor marker across a 20-meter distance within an allotted time frame. The allotted time given to reach the specified distance incrementally shortened as the test progressed. If the student twice failed to reach the other floor marker, the test was terminated [24]. The final score was recorded in laps.

2.3. Procedures. Data were collected at the end of spring semester, 1-3 weeks before the last day of school, and again at the beginning of fall semester, approximately 1-3 weeks after the first day of class. Pedometers were handed out to the homeroom teachers on a Monday morning and then were given to each student. Each pedometer had an identification 
TABLE 1: Mean health-related fitness and pedometer steps for sex groups at spring and fall time-points.

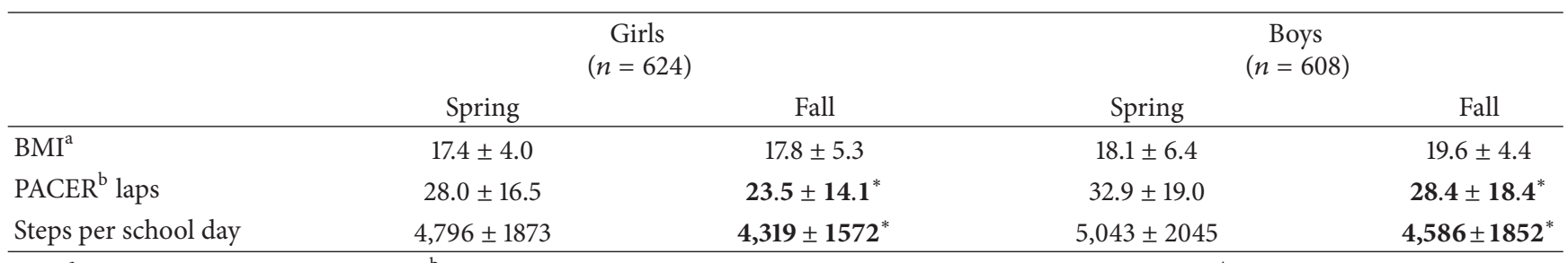

Note. ${ }^{\mathrm{a}} \mathrm{BMI}$ stands for body mass index; ${ }^{\mathrm{b}} \mathrm{PACER}$ stands for the Progressive Aerobic Cardiovascular Endurance Run; ${ }^{*}$ statistically significant difference compared to the spring time-point, $P<0.01$.

number and was given to the student with the corresponding identifier. Before handing the pedometers to each homeroom teacher, each pedometers' accuracy in measuring steps was checked using the "shake" test, using the procedures outlined by Vincent and Sidman [25]. Students put on the pedometers using the aforementioned procedures at approximately 8 a.m. every morning and took off the pedometers at 3 p.m. in the afternoon. The pedometers were not taken off during any time during the school day. Pedometers were collected from the homeroom teachers on Friday and the data were then entered into an Excel spreadsheet.

HRF measures were collected during physical education. Students entered a private screening area to have their height and weight collected. The PACER was administered in sexspecific groups of approximately 8-12 children per group. The PACER was administered indoors at each of the three schools during both time-points. One trained research assistant collected all anthropometric measures and one trained research assistant collected PACER measures at each school to maintain testing consistency.

2.4. Statistical Analysis. Data were screened for outliers using $z$-scores and box plots and checked for Gaussian distributions using $k$-density plots. A $6 \times 2 \times 2$ doubly Multivariate Analysis of Covariance (MANCOVA) was employed to examine the effect of grade level (1st-6th grade), sex (girl, boy), and time (spring and fall) on average school day step counts and PACER laps, adjusting for school and classroom level clustering. Statistically significant multivariate effects were followed by separate univariate Analysis of Covariance (ANCOVA) tests with a Bonferroni alpha level adjustment to protect against potentially inflated Type I error. If statistically significant grade main effect was found, a Bonferroni post hoc test with further alpha level adjustment was used. Cohen's delta $(d)$ determined the effect size and practical significance of each pairwise comparison. Effect sizes were classified as small if $d \leq 0.2$, medium if $d \cong 0.5$, and large if $d \geq 0.8$ [26]. The MANOVA assumption of equality of population covariance matrices was checked using Box's $M$ test. Alpha level was originally set at $P \leq 0.05$ and all analyses were carried out using SPSS v21.0 statistical software package (Armonk, NY, USA).

\section{Results}

The descriptive statistics for the total sample and within each sex group are reported in Table 1 at both spring and fall

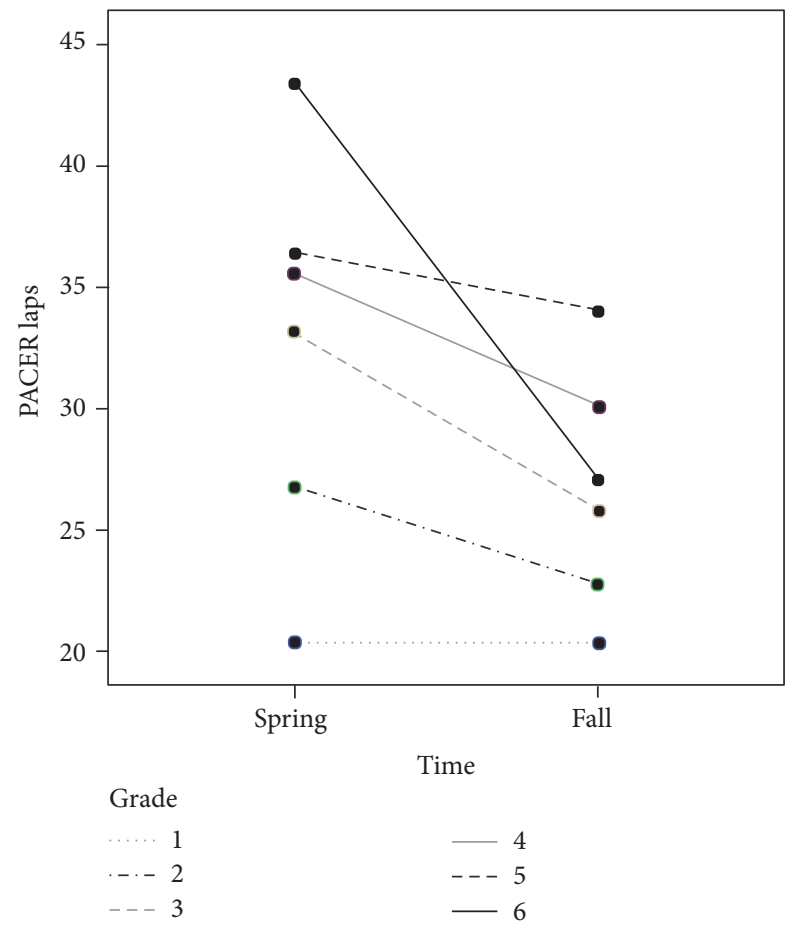

FIGURE 1: Changes in PACER laps between spring and fall timepoints by grade level.

time-points. There were statistically significant multivariate main effects for grade (Wilks' $\Lambda=0.92 ; F=6.3 ; P<0.001$ ), sex (Wilks' $\Lambda=0.99 ; F=3.9 ; P=0.008$ ), and time (Wilks' $\Lambda=0.98 ; F=5.8 ; P<0.001)$ and a statistically significant multivariate grade $\times$ time interaction (Wilks' $\Lambda=0.97 ; F=$ 5.5; $P<0.001)$. Data in Table 1 displays the differences between time-points for health-related fitness (BMI and PACER laps) and school day step counts, respectively. Followup ANOVA tests revealed that the mean pedometer step counts decreased from 4,929 steps in the spring to 4,445 steps in the fall (mean difference $=484$ steps; $P<0.001$; Cohen's $d$ $=0.30$ ) and mean PACER laps decreased from 31.2 laps in the spring to 25.8 laps in the fall (mean difference $=5.4$ laps; $P<$ 0.001 ; Cohen's $d=0.33$ ). Both mean differences represented a small-to-medium sized effect. Follow-up ANCOVA tests also revealed a statistically significant univariate grade $\times$ time interaction for PACER laps $(F=8.2 ; P<0.001)$. Figure 1 is a line plot displaying the change in PACER laps between spring and fall time-points by grade level. Children in the sixth grade 
showed significantly greater decreases between spring and fall time-points in PACER laps compared to all other grade levels $(P<0.001)$ and children in second through sixth grades displayed significantly greater decreases in PACER laps compared to children from the first grade. In particular, the average BMI for girls $(n=624)$ was 17.8 ( $\mathrm{SD}=4.6)$, and average BMI for boys $(n=608)$ was $18.8(\mathrm{SD}=5.4)$. According to CDC's (The Centers for Disease Control and Prevention) BMI-for-age growth charts [27], both girls and boys in this study are averagely classified into the normal weight category. There were no mean differences between the spring and fall time-points on BMI $(P>0.01)$.

\section{Discussion}

The purpose of this study was to examine the effect of a 12week summer break on physical activity and HRF in lowincome children who were enrolled in three CSPAP schools. The results indicated that step counts and PACER laps were lower at the beginning of the fall semester compared to the previous spring semester, prior to a 12 -week summer break. This study provides empirical evidence that any benefits from a CSPAP intervention may be lost when children are out of school. Because data were collected on low-income children, the results do not generalize to higher socioeconomic pediatric populations. Strategies must be devised from personnel working within a CSPAP model to attenuate the declines in physical activity and HRF that may occur during a long break in between school sessions.

An explanation of the CSPAP intervention is needed to communicate the potential benefits these school-based programs may manifest across a school year. CSPAP's primary focus was to provide training and assistance to improve the quality of physical education at each of the three schools. Specifically, monthly in-service opportunities and teacher training were provided to ensure that physical education met national standards, was student-centered, and was developmentally appropriate. The goal was set for teachers to maximize physical activity opportunities through greater student engagement, improved lesson planning, and decreased management and waiting time. Physical education was taught one day per week for 50 minutes and Dynamic Physical Education for Elementary School Children curriculum was employed [28].

During the CSPAP intervention, classroom teachers were asked to implement at least one and encouraged to regularly attempt three-minute activity breaks throughout the day using general activity breaks (including Energizers) or the "TAKE10!" program [29]. Examples of physical activity breaks in the classroom included a stretching or relaxation break, walking around the classroom or hallway, jumping with an invisible jump rope, doing squats, push-ups, sit-ups, and/or passing a ball around the classroom.

In addition to improving the quality of physical education, CSPAP offered physical activity opportunities throughout the school day during recess. Recesses were led by a Physical Activity Leader (PAL) and offered a significant number of opportunities for children to engage in free play or semistructured physical activity while also allowing them to apply the skills learned during their physical education lessons. Each school offered a 15-minute recess immediately following lunch as well as a 15-minute afternoon recess (the length and frequency of recess did not change throughout the school year).

CSPAP is becoming a popular model for increasing children's physical activity levels during the school day. Using the aforementioned behavioral model, in this sample of lowincome children, CSPAP increased average steps per day by approximately 600 steps and increased time in MVPA by approximately 4.0 minutes per day between the beginning and end of the school year (in review). In addition, cardiorespiratory levels increased, on average, by approximately 6.5 PACER laps (in review). Both of these increases represented a small-to-medium sized effect $(d \approx 0.4$; in review). However, as the results from this study have indicated, pedometer step counts decreased by approximately 500 steps per school day and PACER laps decreased by 5.4 laps. Therefore, summer breaks may impose a threat to the long-term efficacy of CSPAP programming in low-income children.

The lower step counts recorded during fall semester compared to spring semester may not totally reflect lower physical activity behaviors during the summer months. The lower step counts displayed in the fall semester in this sample may have reflected the novelty of the school year for both students and staff and the partial implementation of the CSPAP program itself, as teachers may not have fully implemented physical activity breaks, PE lessons may not have commenced, and the PALs at each of the three schools may not have yet devised plans to provide students with physical activity opportunities at recess.

Although the summer months have been shown to be a time period where physical activity levels tend to be higher in children [30,31], some well-designed longitudinal studies have showed that physical activity may actually be compromised during the summer, especially in girls [20], in climates where outdoor temperatures are too hot and uncomfortable for children to consistently participate in active play. Previous study has also suggested that there are decreases in MVPA and increases in sedentary behaviors as school students grow up [32], and these unfavorable trends have been more drastic in girls compared with boys. This explains that girls demonstrated an overall lower PA level than boys in this study. Data in this study were collected from a state from the Mountain West Region of the US, where temperatures are often in the range of 90 to 100 degrees Fahrenheit during the majority of a summer day. Therefore, it is possible that hot and uncomfortable summer temperatures may compromise the physical activity behaviors of low-income children in certain regions of the US. The phenomenon, in addition to the barriers that low-income children face to achieve 60 minutes of MVPA per day, makes it theoretically logical that summer breaks would be significantly detrimental to optimize physical activity and HRF in this pediatric population. Although no mean BMI differences between the spring and fall time-points were found, both girls and boys demonstrated slight increase on their BMI. A plausible explanation is that the children gained their weights during the summer days 
due to the lack of the organized physical education classes, which also echoes the facts that participants' PA levels were decreased during the summer time.

The lower recorded step counts in the fall may reflect decreases in ambulatory physical activity behaviors and thus may have affected cardiorespiratory endurance levels. Cardiorespiratory endurance is an important component to HRF in children and can distinguish, with a reasonable amount of accuracy, children who have unfavorable cardiometabolic biomarkers from healthy children [33]. The average PACER score at the fall time-point was approximately 5 laps fewer compared to the spring time-point. The decrease in cardiorespiratory endurance may be attributable to decreases in ambulatory physical activity during the summer months. Interestingly, first graders in this sample did not experience any mean decrease in PACER laps, while the sixth graders' PACER laps drastically decreased between the two timepoints. At the commencement of adolescence, children's MVPA decreases significantly and this trend tracks throughout the developmental years and into young adulthood [34, 35]. Decreases in MVPA may be accompanied by decreases in cardiorespiratory endurance levels $[35,36]$. Sixth graders in this sample had nearly a 15-lap decrease in PACER laps from spring to fall time-points. If not addressed, low levels of cardiorespiratory endurance may track through adolescence and further influence health risk [37].

The results from this study manifest important practical implications. Even though CSPAP has the potential to increase physical activity and HRF levels in children, breaks in CSPAP may lead to a decline in these constructs and ultimately attenuate any benefits it may have accumulated across a school year. Practitioners and teachers working within the CSPAP model need to be aware of these potential declines after a break from CSPAP programming. Although it is difficult to follow up students during the summer months, devising a physical activity or HRF summer plan for each student within CSPAP schools may facilitate the continuation of CSPAP principles throughout the summer months. Although adherence to the principles is not guaranteed, especially in younger children, it still may partially attenuate potential decreases in physical activity and HRF during long school breaks, especially in those children who do not have many physical activity opportunities during the summer. Physical activity experts and physical education teachers may consider other strategies. Because each school has its own unique characteristics in student composition, geographical area, and proximately to physical activity promoting facilities (e.g., family centers and playgrounds), derivation of physical activity strategies during the summer months needs to be constructed to meet each school's and most specifically to meet each child's needs. It will be advantageous for CSPAP personnel to address the aforementioned considerations prior to the end of the school year.

There are limitations to this study that must be considered before any generalizations can be made. First, the majority of the sample consisted of low-income children who were of an ethnic minority; therefore the external validity of the results is questionable if generalized to other populations of children with different ethnic and/or socioeconomic representation.
Also, the geographical regions may have significantly influenced the results; therefore the external validity of the results is highly questionable if generalized to other geographical regions, especially regions characterized by summers with relatively cooler average temperatures. Also, there was no control group to compare the CSPAP schools with schools that do not employ CSPAP. The internal validity of the results would be stronger if CSPAP schools were compared to nonCSPAP schools. Finally, measurement of physical activity consisted of using pedometers to record step counts. Pedometers do not capture the intensity of ambulatory physical activity and only capture lower-body movements; therefore the construct validity of this instrument is questionable if one were to generalize the results to whole body physical activity of varying intensities. However, because of the large sample size and time constraints for collecting and analyzing the data, pedometers were considered to be the most efficient objective instrument for population-based physical activity surveillance in school settings.

In conclusion, low-income children from CSPAP schools displayed fewer step counts and PACER laps in the fall compared to the previous spring. For cardiorespiratory endurance, older elementary school-aged children showed greater decreases compared to children in the first grade. The results provide empirical evidence suggesting that the improvements accumulated over one year of CSPAP may be partially or fully eliminated after long breaks in school sessions in low-income children. Practitioners and teachers that are involved in CSPAP programs need to be aware of the potential declines in physical activity and HRF levels that may occur after a summer break in low-income children. Strategies must be devised late in the school year to help attenuate these potential declines in physical activity and HRF. Although CSPAP shows promise to improve the health of children, long breaks in between school sessions threaten the long-term efficacy of such programming.

\section{Conflicts of Interest}

The authors declare that they have no conflicts of interest.

\section{Acknowledgments}

This work was supported by a Grant no. S215F140118 from the US Department of Education in the form of a Carol White Physical Education Program (PEP) Grant. The authors would like to thank the US Department of Education for funding this study. The authors would like to thank the schools and students who participated in this study.

\section{References}

[1] L. M. Boddy, M. H. Murphy, C. Cunningham et al., "Physical activity, cardiorespiratory fitness, and clustered cardiometa bolic risk in 10- to 12-year-old school children: the REACH Y6 study," American Journal of Human Biology, vol. 26, no. 4, pp. 446-451, 2014. 
[2] Centers for Disease Control and Prevention, The Association between School-Based Physical Activity, Including Physical Education, and Academic Performance, U.S. Department of Health and Human Services, Atlanta, Ga, USA, 2010.

[3] U.S. Department of Health \& Human Services, Physical Activity Guidelines for Americans (Report), U.S. Department of Health \& Human Services, Washington, DC, USA, 2008.

[4] R. P. Troiano, D. Berrigan, K. W. Dodd, L. C. Mâsse, T. Tilert, and M. Mcdowell, "Physical activity in the United States measured by accelerometer," Medicine and Science in Sports and Exercise, vol. 40, no. 1, pp. 181-188, 2008.

[5] Institutes of Medicine, Educating the Student Body: Taking Physical Education to School, The National Academies Press, Washington, DC, USA, 2013.

[6] Centers for Disease Control and Prevention, Comprehensive School Physical Activity Programs: A Guide for Schools, U.S. Department of Health and Human Services, Atlanta, Ga, USA, 2013.

[7] K. A. Vander Ploeg, J. McGavock, K. Maximova, and P. J. Veugelers, "School-based health promotion and physical activity during and after school hours," Pediatrics, vol. 133, no. 2, pp. e371-e378, 2014.

[8] R. D. Burns, T. A. Brusseau, and J. C. Hannon, "Effect of a comprehensive school physical activity program on school day step counts in children," Journal of Physical Activity and Health, vol. 12, no. 12, pp. 1536-1542, 2015.

[9] M. R. Carnethon, M. Gulati, and P. Greenland, "Prevalence and cardiovascular disease correlates of low cardiorespiratory fitness in adolescents and adults," Journal of the American Medical Association, vol. 294, no. 23, pp. 2981-2988, 2005.

[10] J. C. Eisenmann, G. J. Welk, M. Ihmels, and J. Dollman, “Fatness, fitness, and cardiovascular disease risk factors in children and adolescents," Medicine and Science in Sports and Exercise, vol. 39, no. 8, pp. 1251-1256, 2007.

[11] G. J. Welk, S. B. Going, J. R. Morrow Jr., and M. D. Meredith, "Development of new criterion-referenced fitness standards in the FITNESSGRAM ${ }^{\circledR}$ program," American Journal of Preventive Medicine, vol. 41, no. 4, pp. S63-S67, 2011.

[12] Committee on Fitness Measures and Health Outcomes in Youth, Food and Nutrition Board, Institute of Medicine, R. Pate, M. Oria, and L. Pillsbury, Eds., Fitness Measures and Health Outcomes in Youth, Health-Related Fitness Measures for Youth: Cardiorespiratory Endurance, National Academies Press, Washington, DC, USA, 2012.

[13] Committee on Fitness Measures and Health Outcomes in Youth, Food and Nutrition Board, Institute of Medicine, R. Pate, M. Oria, and L. Pillsbury, Eds., Fitness Measures and Health Outcomes in Youth, Health-Related Fitness Measures for Youth: Body Composition, National Academies Press, Washington, DC, USA, 2012.

[14] I. Ferreira, K. Van Der Horst, W. Wendel-Vos, S. Kremers, F. J. Van Lenthe, and J. Brug, "Environmental correlates of physical activity in youth-a review and update," Obesity Reviews, vol. 8, no. 2, pp. 129-154, 2007.

[15] P. Gordon-Larsen, M. C. Nelson, P. Page, and B. M. Popkin, "Inequality in the built environment underlies key health disparities in physical activity and obesity," Pediatrics, vol. 117, no. 2, pp. 417-424, 2006.

[16] S. E. Messiah, K. L. Arheart, G. Lopez-Mitnik, S. E. Lipshultz, and T. L. Miller, "Ethnic group differences in cardiometabolic disease risk factors independent of body mass index among american youth," Obesity, vol. 21, no. 3, pp. 424-428, 2013.
[17] N. F. Butte, M. R. Puyau, A. L. Adolph, F. A. Vohra, and I. Zakeri, "Physical activity in nonoverweight and overweight hispanic children and adolescents," Medicine and Science in Sports and Exercise, vol. 39, no. 8, pp. 1257-1266, 2007.

[18] A. M. Lampard, J. M. Jurkowski, H. A. Lawson, and K. K. Davison, "Family ecological predictors of physical activity parenting in low-income families," Behavioral Medicine, vol. 39, no. 4, pp. 97-103, 2013.

[19] A. Borraccino, P. Lemma, R. J. Iannotti et al., "Socioeconomic effects on meeting physical activity guidelines: comparisons among 32 countries," Medicine and Science in Sports and Exercise, vol. 41, no. 4, pp. 749-756, 2009.

[20] N. D. Ridgers, J. Salmon, and A. Timperio, "Too hot to move? Objectively assessed seasonal changes in Australian children's physical activity," International Journal of Behavioral Nutrition and Physical Activity, vol. 12, no. 1, 2015.

[21] T. Baranowski, W. O. Thompson, R. H. DuRant, J. Baranowski, and J. Puhl, "Observations on physical activity in physical locations: age, gender, ethnicity, and month effects," Research Quarterly for Exercise and Sport, vol. 64, no. 2, pp. 127-133, 1993.

[22] T. L. Hart, T. Brusseau, P. H. Kulinna, J. J. McClain, and C. Tudor-Locke, "Evaluation of low-cost, objective instruments for assessing physical activity in 10-11-year-old children," Research Quarterly for Exercise and Sport, vol. 82, no. 4, pp. 600-609, 2011.

[23] J. P. Barfield, D. A. Rowe, and T. J. Michael, "Interinstrument consistency of the Yamax Digi-Walker pedometer in elementary school-aged children," Measurement in Physical Education and Exercise Science, vol. 8, no. 2, pp. 109-116, 2004.

[24] M. Meredith and G. J. Welk, Fitnessgram/Actvitygram Test Administration Manual, Human Kinetics, Champaign, Ill, USA, 4th edition, 2010.

[25] S. D. Vincent and C. L. Sidman, "Determining measurement error in digital pedometers," Measurement in Physical Education and Exercise Science, vol. 7, no. 1, pp. 19-24, 2003.

[26] J. Cohen, Statistical Power Analysis for the Behavioral Sciences, L. Erlbaum Associates, Hillsdale, NJ, USA, 1998.

[27] R. J. Kuczmarski, C. L. Ogden, L. M. Grummer-Strawn et al., "CDC growth charts: United States," Advance Data, no. 314, pp. $1-27,2000$.

[28] R. P. Pangrazi and A. Beighle, Dynamic Physical Education Curriculum Guide: Lesson Plans for Implementation, Benjamin Cummings, San Francisco, Calif, USA, 17th edition, 2012.

[29] T. L. Goh, J. Hannon, C. A. Webster, L. W. Podlog, T. Brusseau, and M. Newton, "Effects of a classroom-based physical activity program on children's physical activity levels," Journal of Teaching in Physical Education, vol. 33, no. 4, pp. 558-572, 2014.

[30] P. Tucker and J. Gilliland, "The effect of season and weather on physical activity: a systematic review," Public Health, vol. 121, no. 12, pp. 909-922, 2007.

[31] C. Rich, L. J. Griffiths, and C. Dezateux, "Seasonal variation in accelerometer-determined sedentary behaviour and physical activity in children: a review," International Journal of Behavioral Nutrition and Physical Activity, vol. 9, article 49, 2012.

[32] Y. Fu, Z. Gao, J. C. Hannon, R. D. Burns, and T. A. Brusseau, "Effect of the SPARK program on physical activity, cardiorespiratory endurance, and motivation in middle-school students," Journal of Physical Activity and Health, vol. 13, no. 5, pp. 534542, 2016.

[33] K. R. Laurson, J. C. Eisenmann, and G. J. Welk, "Development of youth aerobic-capacity standards using receiver operating characteristic curves," American Journal of Preventive Medicine, vol. 41, no. 4, pp. S111-S116, 2011. 
[34] R. Telama, X. Yang, E. Leskinen et al., "Tracking of physical activity from early childhood through youth into adulthood," Medicine and Science in Sports and Exercise, vol. 46, no. 5, pp. 955-962, 2014.

[35] A. Raustorp and Y. Ekroth, "Tracking of pedometer-determined physical activity: a 10-year follow-up study from adolescence to adulthood in Sweden," Journal of Physical Activity and Health, vol. 10, no. 8, pp. 1186-1192, 2013.

[36] A. Rauner, F. Mess, and A. Woll, "The relationship between physical activity, physical fitness and overweight in adolescents: a systematic review of studies published in or after 2000," BMC Pediatrics, vol. 13, no. 1, article 19, 2013.

[37] J. W. R. Twisk, H. C. G. Kemper, and W. Van Mechelen, "The relationship between physical fitness and physical activity during adolescence and cardiovascular disease risk factors at adult age. The Amsterdam Growth and Health Longitudinal Study," International Journal of Sports Medicine, Supplement, vol. 23, no. 1, pp. S8-S14, 2002. 


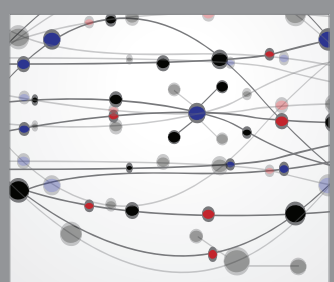

The Scientific World Journal
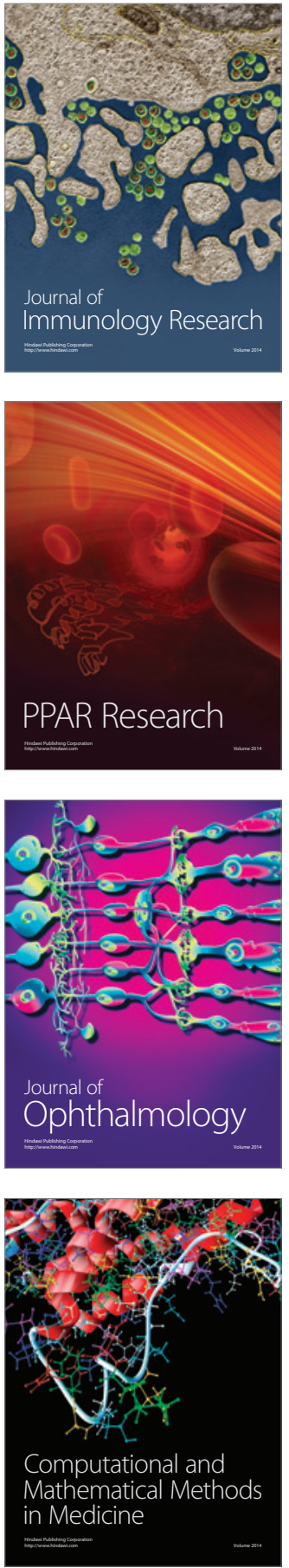

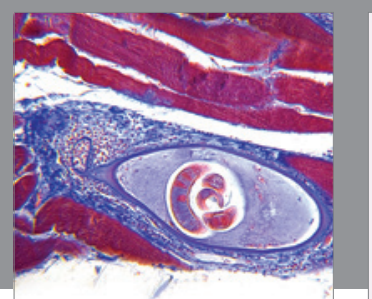

Gastroenterology Research and Practice
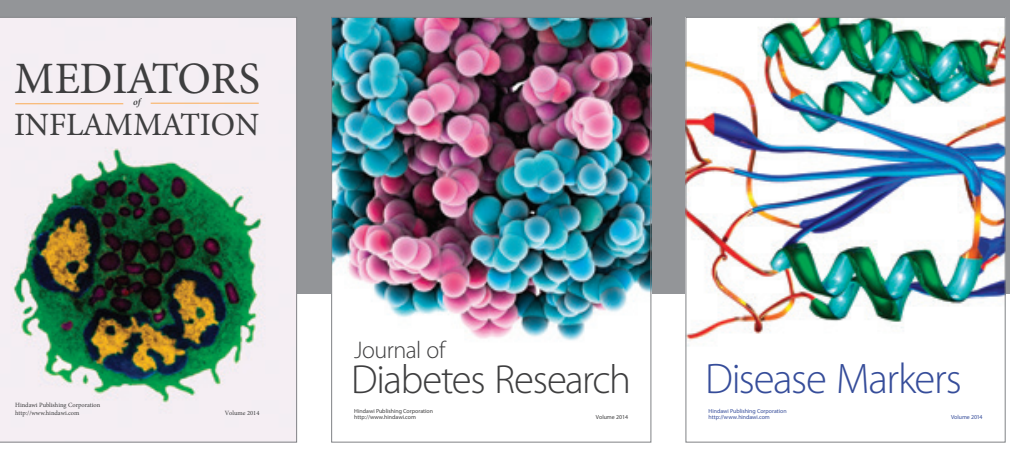

Disease Markers

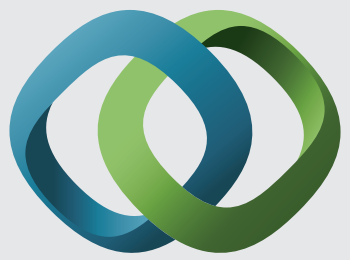

\section{Hindawi}

Submit your manuscripts at

https://www.hindawi.com
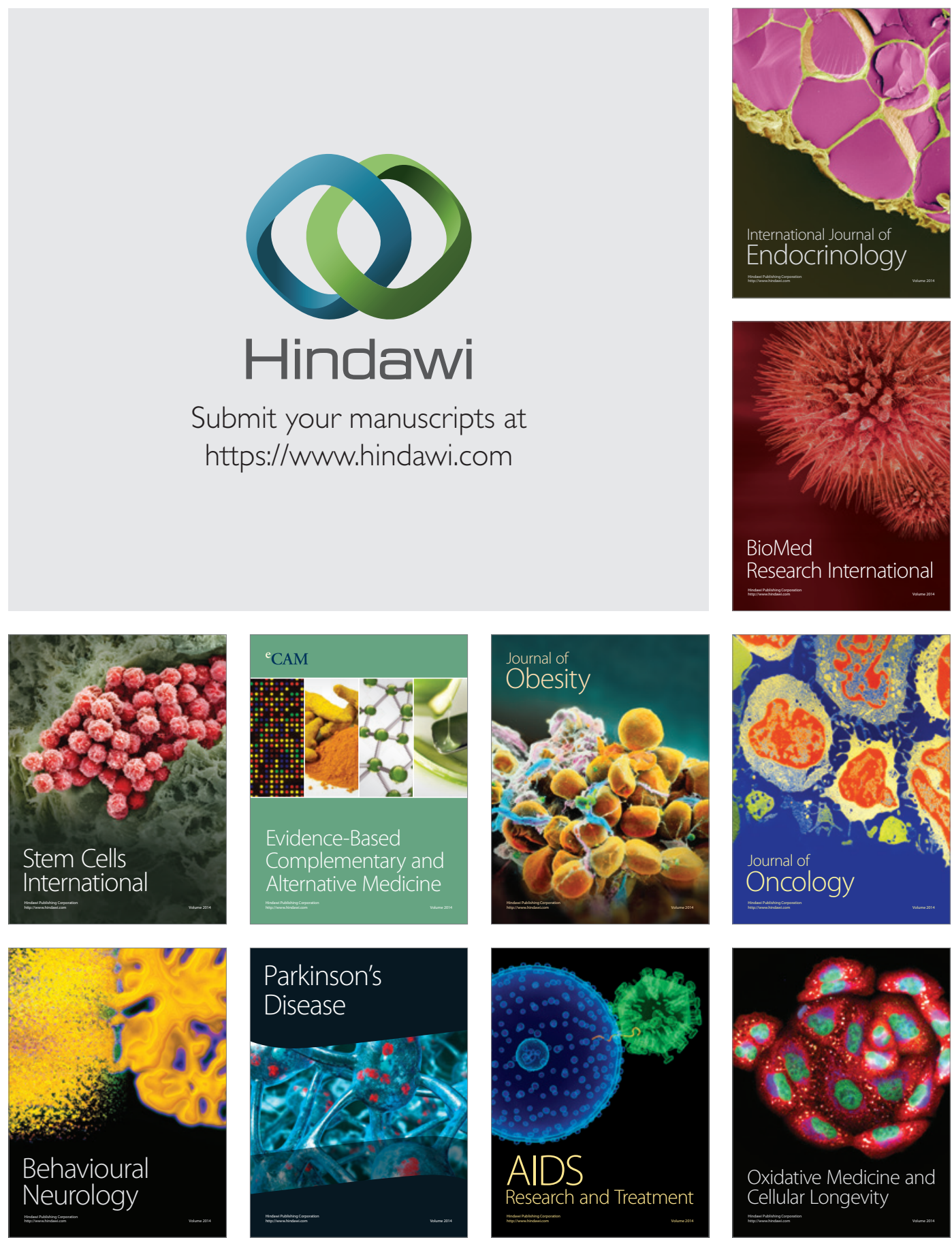